\title{
QUEENS COLLEGE RADIOCARBON MEASUREMENTS I
}

\author{
R PARDI \\ Radiocarbon Laboratory, Queens College, City University of New York, \\ Flushing, New York 11367
}

\section{INTRODUCTION}

The radiocarbon dating laboratory of Queens College, CUNY, was established with the joint support of the Research Foundation of the City University of New York and the departments of Anthropology, Biology, Chemistry, and Earth and Environmental Sciences of Queens College. The laboratory has been operational since June, 1975 and is located in the basement of a two-story concrete and brick building.

A liquid scintillation counter, Picker Liquimat Model 650-220, is employed, adjusted for ${ }^{14} \mathrm{C}$ dating use. Seven vials (5 samples, a sealed background and standard) are cycled at 40 minute intervals for one week or more.

Benzene synthesis from sample $\mathrm{CO}_{2}$ follows that described by Tamers (1965) and Noakes et al (1967). Dry-packed lithium shot is used without prior scrubbing. The vanadia-alumina catalyst (Harshaw Chemicals V-0701 T 1/8) is initially activated as in Coleman et al (1972) prior to use and reactivated as in Polach et al (1972) between samples.

Counting vials are quartz similar in design to those of Polach (1969) but have a polyethylene cap with Teflon $^{\mathrm{R}}$ seal rather than ground glass. Relative backgrounds between vials are found to be a linear function of weight, after correction for counting efficiency. Backgrounds range between ca 5.2 and $6.4 \mathrm{cpm}$ in the ${ }^{14} \mathrm{C}$ channel. All background activity above ca $1.7 \mathrm{cpm}$ can be accounted for by ppm concentrations of potassium in the quartz. Two sealed vials are provided for permanent background and NBS oxalic acid. Cocktail recipe consists of 4cc sample and lcc of benzene-phosphor with resulting concentrations of $0.5 \% \mathrm{PPO}$ and $0.02 \%$ POPOP.

Count rate for 0.95 NBS oxalic acid is $8.595 \pm .045 \mathrm{cpm} / \mathrm{gC}$. Pretreatment procedures follow closely those recommended in Olson (1963).

All results are based on the conventional half-life for ${ }^{14} \mathrm{C}, i e, 5568 \pm$ 30 years. Results are reported in years before 1950. Errors are $1 \sigma$, based on the combined statistical counting error of the sample, background and standard. ${ }^{13} \mathrm{C} /{ }^{12} \mathrm{C}$ measurements and corrections have not been made for these samples.

\section{ACKNOWLEDGMENTS}

Don Haarman, Judy Berry, and Linda Kaplan assisted in sample preparations. The support and encouragement of David $\mathrm{H}$ Speidel and Walter S Newman are especially appreciated. Special thanks to the late Slainte Mhaith Agat for technical advice and assistance in setting up the lab. 


\section{SAMPLE DESGRIPTIONS}

I. GEOLOGIC SAMPLES

QC-100. Bannerman Island
1) $5985 \pm 125$
4035 BC
2) $5920 \pm 115$
3970 BC

Shells (Crassostrea virginica) in small midden, depth .31 to $.62 \mathrm{~m}$, Hudson Highlands Gorge, $98 \mathrm{~km}$ upstream from Narrows in New York City $\left(41^{\circ} 27^{\prime} 28^{\prime \prime} \mathrm{N}, 73^{\circ} 58^{\prime} 55^{\prime \prime} \mathrm{W}\right)$. Coll and subm by W S Newman, Dept Earth and Environmental Sci, Queens College, CUNY, Flushing, New York. Comment (WSN): split of identical sample previously dated, RL-177: $6150 \pm 120$. Charcoal from hearth apparently below shell stratum, M-287: $4480 \pm 300$ (Newman, 1974).

\section{Niagara Falls area series}

Snail and clam shell from Niagara Falls area. Coll and subm by $\mathrm{P}$ Calkin, Dept Geol Sci, SUNY, Buffalo, Amherst, New York.

$$
\begin{aligned}
\text { 1) } 1220 & \pm 75 \\
\text { AD } 730 & \\
\text { 2) } 1240 & \pm 160
\end{aligned}
$$

QC-116. Lake Tonawanda Sand

Snail and clam fragments (unioids) from Laun I. $\left(43^{\circ} 05^{\prime} \mathrm{N}, 79^{\circ} 03^{\prime}\right.$ $\mathrm{W})$, depth $2.5 \mathrm{~m}$ beneath surface, alt $175 \mathrm{~m}$. Comment $(\mathrm{PC})$ : from very fine to fine grained sand of Lake Tonawanda overlying red clay of Glacial Lake Warren or later glacial lake. Previous date, BGS-273: $3780 \pm 90$. Neither date conforms to expected age, $>10,000$.

\section{QC-117. Whirlpool, Niagara River gravel}

Snail shells (Goniabasis sp) from Goat Island site $\left(43^{\circ} 05^{\prime} \mathrm{N}, 79^{\circ} 04^{\prime}\right.$ $\mathrm{W})$, surface, from slump scar just outside railing facing Thompson Point, Ontario, at lower end of Whirlpool. Comment (PC): from old Niagara R gravels at Whirlpool Park, Niagara Falls, New York. Previous date, BGS274: $9770 \pm 150$. Brock date on unioid without acid leaching.

\section{QC-118. Goat Island, Niagara River gravel}

$9115 \pm 215$

Clam-shell fragments (mostly unioid) from Goat Island site, from surface, alt $168 \mathrm{~m}$. Comment $(\mathrm{PC})$ : same as $\mathrm{QC}-117$, gives maximum age for retreat of Falls to Goat I. Previous date, BGS-275: $9080 \pm 130$.

QC-121. East Hampton-Hither Hills Tombolo $\quad \delta^{14} \mathbf{C}=+\mathbf{4 5} \pm \mathbf{2 0} \%$

Shell (Strombus pugilis alatus). Coll and subm by W S Newman. Surface colln from $1 \mathrm{~km} \mathrm{NE}$ of Beach Hampton $\left(49^{\circ} 59^{\prime} 5^{\prime \prime} \mathrm{N}, 72^{\circ} 5^{\prime} 30^{\prime \prime}\right.$ W). Comment (WSN): presently, this species not found N of North Carolina. Date suggests that sample was discarded from a private colln. 


\section{Dogan Point series}

II. ARCHAEOLOGIC SAMPLES

Shells and charcoal from Dogan Point, Montrose Point, New York, Haverstraw quad $\left(41^{\circ} 14^{\prime} 10^{\prime \prime} \mathrm{N}, 73^{\circ} 56^{\prime} 50^{\prime \prime} \mathrm{W}\right)$. Coll and subm by L A Brennan, Briarcliff Coll, Briarcliff Manor, New York.

General Comment (LAB): most shells are Middle Archaic, with some Late Archaic and transitional. Traces of ceramic cultures are present. There is no horizontal stratification and middens are generally weathered and mixed. Site elev $3 \mathrm{~m}$.

Shells (Ostrea), depth $103 \mathrm{~cm}$. Comment (LAB): from basal layer of complex midden ca .4 h. Previous date from overlying shell horizon, L-1038E: $5650 \pm 200$. Shells from 4.5m away, GX-1918: $5155 \pm 135$, and L-1381: $6950 \pm 100$.

\section{QC-103. Dogan Point 3}

Shells (Ostrea), from surface. Comment (LAB): surface disturbed by weather and traffic (wagon, logging). Small shell size.

\section{QC-104. Dogan Point 4}

$$
\begin{array}{r}
310 \pm 65 \\
\text { AD } 1640
\end{array}
$$

Charcoal from hearth, depth 36 to $53 \mathrm{~cm}$. Date indicates mixture of later (colonial?) charcoal with older shells.

\section{QC-105. Dogan Point 5}
1) $4560 \pm 80$ $2610 \mathrm{BC}$

Shell (Ostrea), from surface. Comment (LAB): in contact with Otter Creek type point, previously dated, GX-2324: $5095 \pm$ 150, GX-1919: 5075 \pm 160, GX-1918: $5155 \pm 135$. Does not appear to date Otter Creek point, but some later shell heap.

\section{Piping Rock series}

Shells from middens along Hudson R, Haverstraw quad (41 ${ }^{\circ} 10^{\prime} 40^{\prime \prime}$ $\left.\mathrm{N}, 73^{\circ} 52^{\prime} 30^{\prime \prime} \mathrm{W}\right)$. Coll and subm by L A Brennan. Comment (LAB): middens are collns of some integrity, randomly found in this area. Site elev, $12 \mathrm{~m}$.

\section{QC-106. Piping Rock 6}

Shell (Ostrea), depth $5 \mathrm{~cm}$. Comment (LAB): previous date of same heap, GX-3371: $3750 \pm 150$. Stratum below, of unusually large shell, was dated, GX-2999: $4730 \pm 140$. 
QC-107. Piping Rock 7

Shells (Ostrea sp), from surface. Comment (LAB): date indicates shells are not assoc with Middle Woodland Fox Creek-Cary material of AD 450 to 600. Shells adjacent to heap dated, GX-3371: $3750 \pm 150$.

\section{QC-108. Piping Rock 8}

$5175 \pm 125$

Shells (Ostrea sp), from discrete midden heap. Comment (LAB): long, narrow shape of shell may denote period of quick silting, with no rise in sea level. Previous date from similar heap, GX-3238: $5135 \pm 155$.

QC-109. Pear Tree 9

$4750 \pm 140$

Shells (Ostrea sp), Pear Tree loc, depth $90 \mathrm{~cm}$ in old beach sand. Comment (LAB): apparent stray from midden containing QC-108.

1) $5110 \pm 100$

$3160 \mathrm{BC}$

QC-110. Cedar Loc 10

2) $4955 \pm 110$

3005 BC

Shell (Ostrea sp), Cedar loc, depth $76 \mathrm{~cm}$. Comment (LAB): from thin lens in beach sand. Same situation as QC-109.

\section{QC-113. Pear Tree 13}

$4735 \pm 115$

Shells (Ostrea sp), depth $66 \mathrm{~cm}$. Comment (LAB): shell from similar level ca $4.5 \mathrm{~m}$ away dated, GX-3238: $5135 \pm 155$. Dates small Beckman or Hudsonbrook point.

\section{Tinsley site series, New Mexico}

Wood and charcoal from Feature A, large circular structure, from posts along S wall, Las Vegas quad $\left(35^{\circ} 31^{\prime} 14^{\prime \prime} \mathrm{N}, 105^{\circ} 13^{\prime} 0^{\prime \prime} \mathrm{W}\right)$, depth 35 to $40 \mathrm{~cm}$. Coll and subm by R Mishler, New Mexico Highlands Univ, Las Vegas, New Mexico.

General Comment (RM): age estimate based on architectural analysis ca AD 1100.

QC-119. TN-A-35-H-6

$$
\begin{array}{r}
\text { 1) } 970 \pm 75 \\
\text { AD } 980 \\
\text { 2) } 890 \\
\text { AD } 1060
\end{array}
$$

Charcoal, preserved with paraffin immediately after colln. Sample given only normal $\mathrm{HCl}, \mathrm{NaOH}$ pretreatment. 
1) $1020 \pm 70$

AD 930

QC-120. TN-A-154-H-1

2) $1035 \pm 70$ AD 915

Charred wood. Paraffin preservative extracted from sample using petroleum ether with ca $30 \mathrm{~min}$ boiling followed by normal $\mathrm{HCl}, \mathrm{NaOH}$ pretreatment. Dates on same material without ether extraction: 1) 1885 $\pm 100,2) 1950 \pm 100$.

\section{QC-128/9. Minisink site}

$1670 \pm 95$

Charcoal from Minisink site, Sqs 8 \& 13, Levels 10 \& 11 , Minisink Hills, Pennsylvania $\left(40^{\circ} 59^{\prime} 34^{\prime \prime} \mathrm{N}, 75^{\circ} 07^{\prime} 59^{\prime \prime} \mathrm{W}\right)$, depth 1.7 to $1.8 \mathrm{~m}$. Coll by E McDowell; subm by C McNett, Upper Delaware Early Man project, American Univ, Washington DC. Comment (CMcN): from shallow pits in Early Archaic zone. Far too young, and indicates that sample occurred at base of intrusive (Woodland) pit, top portion of which was unrecognizable. Exactly matches a date, W-3135: $1640 \pm 200$, from a similar situation in $\mathrm{Sq} 7$, Level 8. This zone should have dated no younger than 5500 BC.

REFERENCES

Coleman, D D, Liu, C L, Dickerson, D R, and Frost, R R, 1972, Improvement in trimerization of acetylene to benzene for radiocarbon dating with commercially available vanadium oxide catalyst: 8th internatl conf on radiocarbon dating, Proc, Lower Hutt, New Zealand, v 1, p 158-170.

Newman, W S, 1974, A comment on Snow's 'Rising Sea Level and Prehistoric Cultural Ecology in Northern New England': Am Antiquity, v 39, p 135-136.

Noakes, J E, Kim, S M, and Akers, L K, 1967, Recent improvements in benzene chemistry for radiocarbon dating: Geochim et Cosmochim Acta, v 13, p 1094-1096.

Olson, E A, 1963, The problem of sample contamination in radiocarbon dating: PhD thesis, Columbia Univ, New York, 320 p.

Polach, H A, 1969, Optimisation of liquid scintillation radiocarbon age determinations and reporting of ages: Atomic Energy in Australia, v 12, p $21-28$.

Polach, H A, Gower, J, and Fraser, I, 1972, Synthesis of high purity benzene for radiocarbon dating: 8th internatl conf on radiocarbon dating, Proc, Lower Hutt, New Zealand, v 1, p 145-147.

Tamers, M A, 1965, Routine ${ }^{14} \mathrm{C}$ dating using liquid scintillation technique: 6 th internatl conf radiocarbon and tritium dating, Proc, Pullman, Washington, p 53-68. 